\title{
Helmintiasis y Eimeriosis Gastrointestinal en Alpacas Criadas al Pastoreo en Dos Granjas Comunales de la Región Pasco, Perú, y su Relación con el Peso y Condición Corporal
}

\author{
Gastrointestinal Helminthiasis and Eimeriosis in Grazing Alpacas in Two \\ Communal Farms of Pasco, Peru and their Relationship with Body Weight \\ AND Body Condition
}

Marine Masson', Gustavo Gutiérrez², Víctor Puicón ${ }^{3}$, Daniel Zárate ${ }^{3,4}$

\section{Resumen}

El objetivo del presente trabajo fue estudiar la relación entre los parásitos gastrointestinales con peso vivo y condición corporal en alpacas criadas al pastoreo en la región altoandina de Pasco, Perú. Se recolectaron muestras de heces de 160 alpacas de dos granjas comunales que fueron analizadas mediante las técnicas de McMaster modificado y coprocultivo para determinar la carga parasitaria y la identificación de las especies parasitarias. Se encontró una baja prevalencia de nematodos, de modo que se decidió estudiar la relación entre la carga parasitaria de Eimeria macusaniensis con los dos parámetros productivos. Los resultados muestran una correlación negativa y significativa entre carga parasitaria y peso vivo $\left(\mathrm{r}^{2}=0.8938\right)$ y una correlación no significativa entre carga parasitaria y condición corporal $\left(\mathrm{r}^{2}=0.5747\right)$.

Palabras clave: alpacas, Eimeria macusaniensis, nematodos, parásitos, Pasco, peso

\section{Abstract}

The aim of this study was to study the relationship between gastrointestinal parasites with body weight and body condition in alpacas grazing in the Andean region of Pasco, Peru. Faecal samples from 160 alpacas in two communal farms were collected and analysed by the McMaster technique and faecal culture to determine the parasite load and parasite species. Due to the low prevalence of nematodes was decided to evaluate the relationship between Eimeria macusaniensis burden with the two productive parameters. The results

\footnotetext{
${ }^{1}$ Ecole Nationale Veterinaire de Toulouse, Francia

${ }^{2}$ Programa de Ovinos y Camélidos Americanos, ${ }^{3}$ Laboratorio de Parasitología, Facultad de Zootecnia, Universidad Nacional Agraria La Molina, Lima, Perú

${ }^{4}$ E-mail:dazre@lamolina.edu.pe
}

Recibido: 24 de marzo de 2016

Aceptado para publicación: 6 de junio de 2016 
showed a negative and significant correlation between parasite load and body weight $\left(\mathrm{r}^{2}=0.8938\right)$ and non-significant correlation between parasite load and body condition $\left(\mathrm{r}^{2}=0.5747\right)$.

Key words: alpacas, Eimeria macusaniensis, nematodes, parasites, Pasco, body weight

\section{INTRODUCCIÓN}

La ganadería alpaquera en comunidades altoandinas del Perú representa una actividad socioeconómica importante, ya que las alpacas representan una fuente de carne, cuero y fibra de considerable valor comercial; además, por su adaptabilidad, son de los pocos animales domésticos que pueden ser explotados comercialmente sobre altitudes superiores a $4000 \mathrm{msnm}$. Sin embargo, diversas enfermedades parasitarias pueden desarrollarse en estas condiciones de crianza, de allí que se les considera como un serio problema sanitario (Ballweber, 2009).

Los parásitos gastrointestinales identificados en alpacas incluyen nematodos, trematodos, cestodos y protozoarios. En Sudamérica se han reportado parásitos específicos para camélidos, como los nematodos Graphinema aucheniae, Spiculopteragia peruviana y Nematodirus lamae, así como parásitos protozoarios del genero Eimeria en el tracto gastrointestinal (Cebra et al., 2014). Las especies del género Eimeria que infectan a alpacas incluyen a $E$. lamae, $E$. macusaniensis, E. ivitaensis, E. punoensis y $E$. alpacae, y son altamente específicos de esta especie (Leguía y Casas, 1999).

Por lo general, en áreas donde los camélidos son criados al pastoreo en forma tecnificada y sin contacto con otras especies domésticas, se pueden evidenciar altas preval encias de Eimeria spp, a diferencia de la crianza mixta, en la cual pueden hallarse múltiples especies parasitarias que son compartidas con otras especies domésticas (Cebra et al., 2014).
Las especies de Eimeria varían en su patogenicidad. E. macusaniensis como agente único o asociado con $E$. lamae o $E$. ivitaensis es considerada como altamente patógena (Cafrune et al., 2009). La asociación de estas especies es sinérgica, generando la destrucción del epitelio intestinal luminal, conllevando a una enteritis y diarrea (Cheney y Allen, 1989), mermando la ganancia de peso $\mathrm{y}$, en ocasiones, causando la muerte, estando su severidad asociada al número de ooquistes ingeridos y a la inmunidad de las alpacas (Cebra et al., 2007).

La eimeriosis afecta principalmente a las crías, causando infecciones de tipo subclínica durante los primeros tres meses de vida, con prevalencias de 30 al 100\% (Leguía y Casas, 1999). Estudios realizados en la sierra sur del Perú reportan altos niveles de infección de $E$. lamae y $E$. macusaniensis en crías de alpacas en comunidades de Puno (Rodríguez et al., 2012) y Cusco (Pérez et al., 2014), mientras que se hallaron altas prevalencias de helmintos pero con cargas parasitarias leves en alpacas jóvenes (Contreras et al., 2014). Sin embargo, no existen reportes sobre el efecto productivo de estas cargas parasitarias en estos animales.

El objetivo del presente estudio fue determinar la prevalencia y carga de helmintiasis y eimeriosis gastrointestinal en alpacas criadas al pastoreo en dos granjas comunales de la región Pasco y estudiar la relación entre la carga parasitaria con el peso vivo y condición corporal en alpacas (Idika et al., 2012; Morales et al., 2012), a fin de determinar el real impacto productivo de estos parásitos en esta importante especie ganadera. 


\section{Materiales y Métodos}

\section{Lugar de Estudio y Animales}

El estudio se realizó en la cooperativa comunal San Pedro de Racco y en la granja comunal de Vicco, localizadas en la región de Pasco, Perú, sobre una altitud de 4300 msnm y con una temperatura promedio anual máxima y mínima de 12.4 y $0.6{ }^{\circ} \mathrm{C}$, respectivamente. La época de lluvias ocurre entre los meses de noviembre y marzo. El estudio se realizó en el mes de julio de 2013.

Las alpacas en estas ganaderías estaban distribuidas en las categorías de plantel y majada, acorde a su mérito genético. En cada lugar se seleccionaron 80 alpacas hembras de raza Huacaya (40 adultas entre 2 y 4 años, y 40 crías de 5 a 6 meses de edad), clasificadas de acuerdo a su dentición.

\section{Muestreo}

Los animales fueron identificados y en el momento del muestreo fueron pesados utilizando una balanza digital suspendida. Además, se determinó la condición corporal en una escala de 1 a 5 (1: muy delgado, 5: sobrepeso), similares a las utilizadas en otras especies (Idika et al., 2012), como indicador del estado de reservas de grasa y del balance energético. Las áreas clave para evaluar la condición corporal fueron el área del lomo justo detrás de las costillas y en frente de la pelvis.

Muestras de heces fueron colectadas directamente del recto del animal (5-10 g), conservadas en cajas isotérmicas de poliestireno expandido con geles refrigerantes, y trasladadas al Laboratorio de Parasitología de la Facultad de Zootecnia, Universidad Nacional Agraria La Molina, Lima.

\section{Análisis Coprológico}

Se utilizó el método de McMaster modificado (Rojas, 2004). Asimismo, se realizó un coprocultivo con las muestras fecales que presentaron huevos tipo Strongílido, recolectándose las larvas de tercer estadio para la determinación de la especie o género mediante el método de Baermann (Rojas, 2004) y su posterior identificación mediante la medición de características larvarias a través de la captura digital y procesamiento de imágenes utilizando el programa Leica Application Suite v. 4.0 .

\section{Análisis Estadístico}

Los resultados fueron expresados como porcentaje de prevalencia del número de alpacas infectadas con ooquistes, huevos tipo strongílido y huevos de nematodos. Además, se determinó la media de cada parásito por gramo de heces en las alpacas infectadas y para cada especie parasitaria se realizó el cálculo de la proporción de larvas de tercer estadio. Para determinar la relación entre carga parasitaria, peso y condición corporal se aplicó un análisis de regresión y de correlación lineal mediante el software estadístico $\mathrm{R}$ y la prueba de Chi-cuadrado como prueba de hipótesis.

\section{Resultados}

De las 160 muestras de heces analizadas, $28.1 \%(\mathrm{n}=45)$ fueron positivas para huevos de tipo strongílido, resultado considerado como prevalencia baja. Asimismo, 41.9\% $(\mathrm{n}=67)$ fueron positivas para ooquistes de $E$. macusaniensis, $26.3 \%(\mathrm{n}=42)$ para huevos de Nematodirus spp, 20.0\% $(\mathrm{n}=32)$ para Trichuris spp y prevalencias menores para otros parásitos (Cuadro 1). El único parásito que mostró una presencia alta fue $E$. macusaniensis, especialmente en crías 
Cuadro 1. Prevalencias ${ }^{1}$ de ooquistes de Eimeria spp y huevos de nematodos gastrointestinales en alpacas en la región de Pasco, Perú (2013)

\begin{tabular}{|c|c|c|c|c|c|c|c|c|c|c|c|c|c|c|c|}
\hline & \multirow[t]{2}{*}{ N. ${ }^{\circ}$} & \multicolumn{2}{|c|}{ HTS } & \multicolumn{2}{|c|}{ Nematodirus } & \multicolumn{2}{|c|}{ Trichuris. } & \multicolumn{2}{|c|}{ Capillaria } & \multicolumn{2}{|c|}{$\begin{array}{c}\text { Lamanema } \\
\text { chavezi }\end{array}$} & \multicolumn{2}{|c|}{$\begin{array}{c}\text { E. macusa- } \\
\text { niensis }\end{array}$} & \multicolumn{2}{|c|}{$\begin{array}{c}E . \\
\text { ivitaensis }\end{array}$} \\
\hline & & $\%$ & HPG & $\%$ & HPG & $\%$ & HPG & $\%$ & $\mathrm{HPG}$ & $\%$ & HPG & $\%$ & $\mathrm{OPG}$ & $\%$ & OPG \\
\hline \multicolumn{16}{|l|}{ Granjas } \\
\hline Racco & 80 & 13.8 & 68 & 18.8 & 67 & 12.5 & 60 & 2.5 & 50 & 5.0 & 63 & 41.3 & 1185 & 5.0 & 750 \\
\hline Vicco & 80 & 42.5 & 118 & 33.8 & 130 & 27.5 & 91 & 7.5 & 67 & 2.5 & 75 & 42.5 & 765 & 8.8 & 1450 \\
\hline \multicolumn{16}{|l|}{ Categoría } \\
\hline Plantel & 80 & 35.0 & 104 & 36.3 & 109 & 23.8 & 71 & 0 & 0 & 5.0 & 63 & 46.3 & 1173 & 6.3 & 1810 \\
\hline Majada & 80 & 21.3 & 103 & 16.3 & 104 & 16.3 & 96 & 10.0 & 63 & 2.5 & 75 & 37.5 & 724 & 7.5 & 683 \\
\hline \multicolumn{16}{|l|}{ Edad } \\
\hline Adultos & 80 & 28.8 & 113 & 7.5 & 67 & 6.3 & 50 & 6.3 & 60 & 3.8 & 67 & 2.5 & 200 & 1.3 & 50 \\
\hline Crías & 80 & 27.7 & 93 & 45.0 & 114 & 33.8 & 87 & 3.8 & 67 & 3.8 & 67 & 81.3 & 995 & 12.5 & 1310 \\
\hline Total & 160 & 28.1 & 103 & 26.3 & 107 & 20.0 & 81 & 5.0 & 63 & 3.8 & 67 & 41.9 & 972 & 6.9 & 1196 \\
\hline
\end{tabular}

${ }^{1}$ HTS: huevo tipo Strongylus; \%: porcentaje de infectados; HPG: media de huevos por gramo de heces; OPG: media de ooquistes por gramo de heces

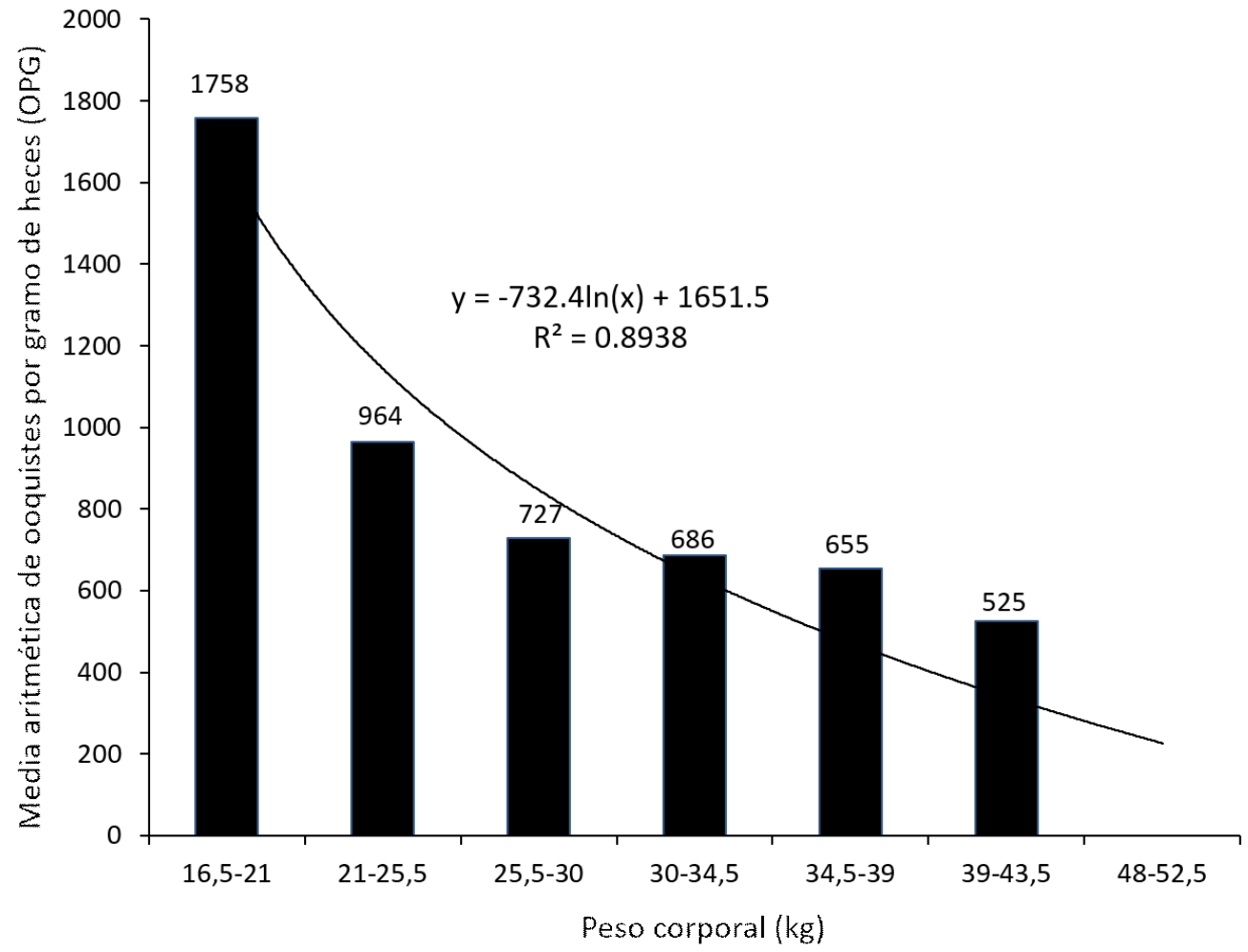

Figura 1. Relación entre la carga de ooquistes de Eimeria macusaniensis y peso corporal en crías de alpacas en la región Pasco, Perú (2013) 


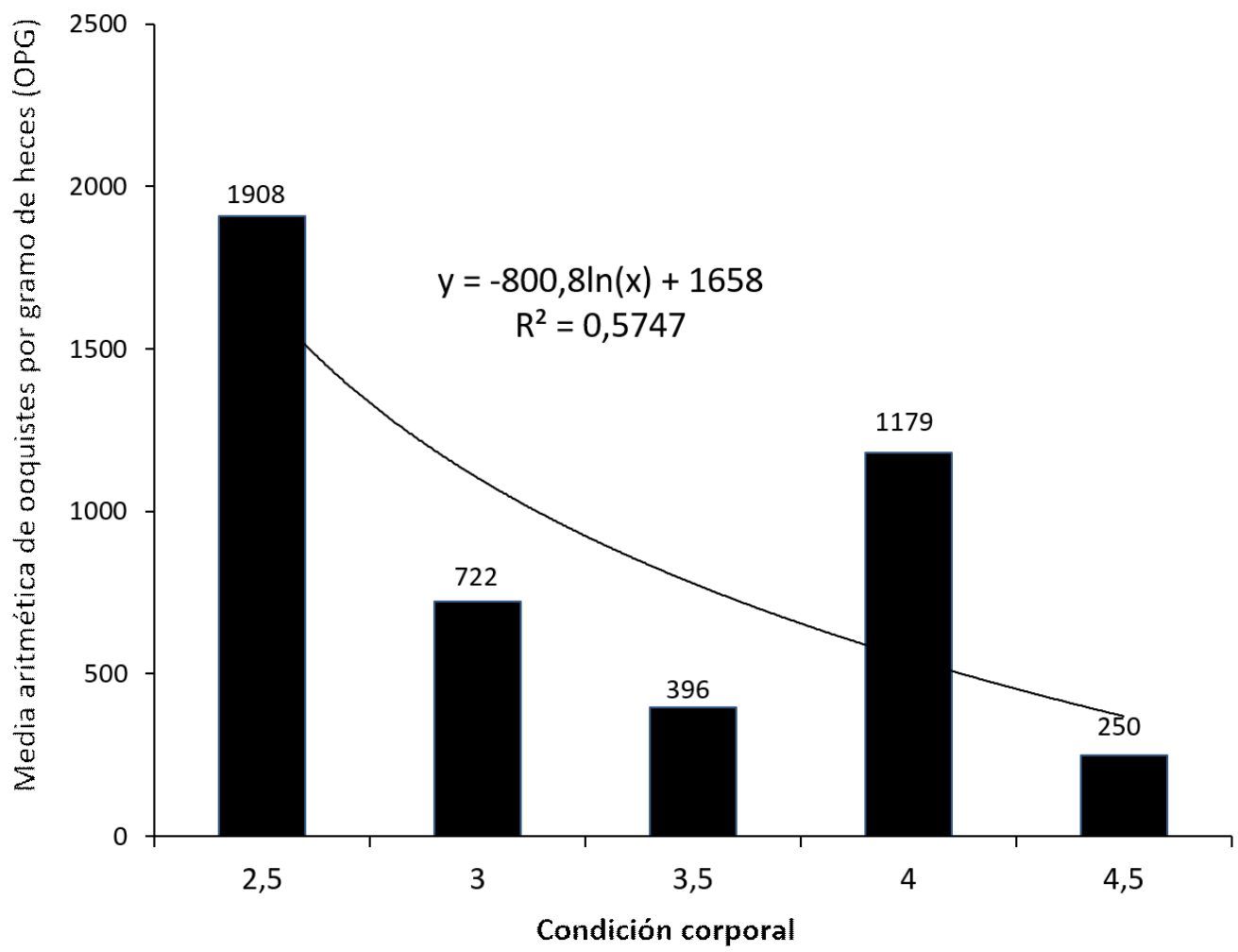

Figura 2. Relación entre la carga Eimeria macusaniensis y condición corporal en crías de alpacas en la región Pasco, Perú (2013)

$(81.3 \%)$ y con un alto recuento fecal $(995$ ooquistes por gramo de heces en promedio). Con estos resultados, se decidió estudiar la posible relación entre el número de ooquistes de E. macusaniensis en crías y su estado corporal.

En general, los parásitos identificados en animales adultos de majada y plantel fueron los mismos: Cooperia spp, Oesophagostomum spp, Teladorsagia circumcincta y Ostertagia ostertagi. Solo Trichostrongylus spp estuvo presente en animales de majada y no en plantel. La predominancia de estas especies era casi siempre en las mismas proporciones, siendo $O$. ostertagi ( $38 \%$ en plantel y $50 \%$ en majada) y en menor poporción
Cooperia spp, Nematodirus spp y $T$. circumcincta.

También se encontró L. chavezi en animales de plantel y Oesophagostomum spp en animales de majada. Si se comparan las especies de larvas presentes en hembras adultas y crías, se puede indicar que las proporciones son diferentes, donde Nematodirus spp solo estuvo presente en crías. En relación con las proporciones de especies larvarias en Racco, se hizo evidente que los animales de plantel y de majada no tuvieron la misma fauna parasitaria, donde $O$. ostertagi fue la especie más común $(60 \%)$ en los adultos de plantel, mientras que Cooperia spp estuvo presente en mayor porcentaje $(63 \%)$ en los adultos de majada. 
En la Figura 1 se observa que las crías con carga alta de E. macusaniensis presentan un menor peso corporal, siendo una correlación inversa y significativa $(\mathrm{p}<0.05)$ y con un alto índice del coeficiente de variabilidad $\left(r^{2}=0.8938\right)$, que es explicada por el modelo propuesto; es decir, el peso corporal refleja la carga parasitaria del parásito.

La Figura 2 muestra, en forma similar, el número de ooquistes de E. macusaniensis según la condición corporal, donde se observa una tendencia no significativa, una baja correlación y un bajo índice del coeficiente de variabilidad $\left(r^{2}=0.5747\right)$, indicando que la relación entre estos parámetros no se ajusta al modelo propuesto, ya que a diferencia del peso corporal, la evaluación de la condición corporal es un parámetro relativamente subjetivo.

\section{Discusión}

Los resultados obtenidos evidencian una carga parasitaria baja de huevos tipo Strongílido en las alpacas de las dos granjas; sin embargo, un recuento bajo no necesariamente significa que existe un mínimo número de nematodos en el animal (Ueno y Goncalves, 1998), ya que se debe considerar factores como sensibilidad de la técnica de Mac Master (50 hpg) y época del año (Bosch y Supperer, 1977), ya que las alpacas fueron evaluadas durante la estación seca; es decir, entre mayo y agosto, periodo que corresponde a la presencia de condiciones hostiles para los nematodos (Leguía y Casas, 1999), pudiendo su presencia no ser detectada debido a mecanismos de sobrevivencia como la hipobiosis (Quiroz, 2005).

La relación entre el número de alpacas infectadas y el lugar de muestreo fue estadísticamente significativa. Las alpacas criadas en Vicco albergan más nematodos que aquellas criadas en Racco. Por el contrario, no existe diferencia significativa con respecto a E. macusaniensis y E. ivitaensis.
Pueden existir diferencias en el manejo de los rebaños entre las granjas que están influyendo en la incidencia de estos parásitos. Además, el sobrepastoreo en Vicco, sumado al comportamiento de pastoreo y los hábitos de defecación de las alpacas, son factores de importancia en el parasitismo gastrointestinal (Bustinza, 2000).

Si las alpacas son criadas junto a ovinos, se favorece un aumento de la infección como la producida en Vicco (Keyyu et al., 2006). Estas dos razones permiten comprender que las alpacas en Vicco son hospederos más frecuentes de nematodos que en Racco. En tanto, el número de infectados con Eimeria spp es similar en ambas granjas, probablemente debido a que las eimerias son parásitos ubicuos en el medio ambiente y naturalmente infectan a la mayoría de los animales en algún grado (Rodríguez et al., 2012).

Se pudo observar que la edad y el número de alpacas infectadas con strongílidos fueron variables independientes; sin embargo, estos criterios fueron dependientes para la infección con Nematodirus spp y Trichuris spp. Las crías son más infectadas con estos parásitos que los animales adultos; además, se puede inferir que las crías de alpacas presentan un mayor número de $E$. macusaniensis e E. ivitaensis que las alpacas adultas. La crianza en conjunto de crías y adultos favorece la infección de las crías, ya que estas son muy susceptibles debido a que su inmunidad aún está en proceso de maduración (Leguía y Casas, 1999). Por otro lado, los adultos estuvieron tan infectados con strongílidos como las crías en San Pedro de Racco y en Vicco, lo cual demuestra que no existió una influencia significativa de la edad.

La relación inversa y correlación significativa $\left(\mathrm{r}^{2}=0.8938\right)$ entre la carga parasitaria de E. macusaniensis y el peso de las crías revela que crías con bajo peso excretaban mayor número de ooquistes. Por otra parte, los recuentos fecales de ooquistes en animales adultos ( 2 a 4 años de edad) fueron nulos. Esto probablemente debido a la característi- 
ca de las coccidias, que tienden a tener una baja frecuencia a nivel del recuento fecal en animales adultos (Rodríguez et al., 2012).

La condición corporal refleja el estado nutricional de un animal, y es importante considerar que animales con baja condición corporal son más susceptibles a los parásitos gastrointestinales (Idika et al., 2012). Por lo tanto, existen muchos factores a considerar como para determinar si la condición corporal en la alpaca es influenciada por el parasitismo gastrointestinal, ya que se encontró una baja relación entre estos parámetros.

\section{Conclusiones}

- Las crías presentaron prevalencias más altas que los adultos.

- La correlación entre peso corporal de crías de alpacas y la carga de Eimeria macusaniensis revela que crías con bajo peso corporal presentan mayor nivel de infección.

- La condición corporal evidenció una tendencia no significativa hacia una relación inversa.

\section{Agradecimiento}

Los autores agradecen a la cooperativa comunal San Pedro de Racco y granja comunal Vicco por el apoyo brindado. El estudio se ejecutó bajo el financiamiento del Proyecto VLIR UOS-UNALM Proyecto 1, Sub-proyecto 1-6 Parasitología en rumiantes y camélidos domésticos en la Sierra Central del Perú.

\section{Litteratura Cittada}

1. Ballweber LR. 2009. Coccidiosis in food animals. In: Smith BP (ed). Large animal internal medicine. St. Louis, USA: Mosby Elsevier. p 1645-1647.
2. Bosch J, Supperer R. 1977. Parasitología en medicina veterinaria. Buenos Aires: Ed Hemisferio Sur. 627 p.

3. Bustinza JA. 2000. Enfermedades de alpacas. $2^{\circ}$ ed. Arequipa: Universidad Nacional del Altiplano. $346 \mathrm{p}$

4. Cafrune MM, Marin RE, Rigalt FA, Romero SR, Aguirre DH. 2009. Prevalence of Eimeria macusaniensis and Eimeria ivitaensis in South American camelids of Northwest Argentina. Vet Parasitol 162: 338-341. doi: 10.1016/j.vetpar.2009.03.006

5. Cheney JM, Allen GT. 1989. Parasitism in llamas. Vet Clin North Am Food Anim Pract 5: 217-225.

6. Cebra CK, Anderson D, Tibary A, Van Saun R, Johnson LW. 2014. Parasitic gastroenteritis. In: Llama and alpaca care. Medicine, surgery, reproduction, nutrition, and health care. St. Louis, MO, USA: Saunders Elsevier. p 501-512.

7. Cebra CK, Valentine BA, Schlipf JW, Bildfell RJ, McKenzie E, Waitt LH, Heidel JR, et al. 2007. Eimeria macusaniensis infection in 15 llamas and 34 alpacas. J Am Vet Med Assoc 230: 94-100. doi: 10.2460/javma.230.1.94

8. Contreras $N$, Chávez A, Pinedo R, Leyva V. Suárez F. 2014. Helmintiasis en alpacas (Vicugna pacos) de dos comunidades de Macusani, Puno, durante la época seca. Rev Inv Vet Perú 25: 268 275. doi: 10.15381/rivep.v25i2.8499

9. Idika IK, Chiejina SN, Mhomga LI, Nnadi PA, Ngongeh LA. 2012. Changes in the body condition scores of Nigerian West African Dwarf sheep experimentally infected with mixed infections of Haemonchus contortus and Trichostrongylus colubriformis. Vet Parasitol 188: 99-103. doi: 10.1016/ j.vetpar.2012.02.020.

10. Кеууи JD, Kassuku AA, Msalilwa LP, Monrad J, Kyvsgaard NC. 2006. Cross sectional prevalence of helminth infections in cattle on traditional, small scale and large scale dairy farms in Iringa district, Tanzania. Vet Res Commun 30: 45-55. 
11. Leguía P, Casas E. 1999. Enfermedades parasitarias y atlas parasitológico de camélidos sudamericanos. Lima: Ed de Mar. $190 \mathrm{p}$.

12. Morales G, Pino L, Sandoval E, Jiménez D, Morales J. 2012. Relación entre la condición corporal y el nivel de infestación parasitaria en bovinos a pastoreo como criterio para el tratamiento antihelmíntico selectivo. Rev Inv Vet Perú 23: 80-89. doi: 10.15381/ rivep.v23i1.886

13. Pérez H, Chávez A, Pinedo R, Leyva V. 2014. Helmintiasis y eimeriasis en alpacas de dos comunidades de Cusco, Perú. Rev Inv Vet Perú 25: 245-253. doi: 10.15381/rivep.v25i2.8497
14. Quiroz H. 2005. Parasitología y enfermedades parasitarias en animales domésticos. México: Limusa. $870 \mathrm{p}$.

15. Rodríguez A, Casas E, Luna L, Gavidia C, Zanabria V, Rosadio $R$. 2012. Eimeriosis en crías de alpacas: prevalencia y factores de riesgo. Rev Inv Vet Perú 23: 289-298. doi: 10.15381/ rivep.v23i3.911

16. Rojas CM. 2004. Nosoparasitosis de los rumiantes domésticos peruanos. $2^{\circ} \mathrm{ed}$. Lima: Ed Maijosa. 383 p.

17. Ueno H, Goncalves PC. 1998. Manual para diagnóstico das helmintoses de ruminantes. $4^{\text {th }}$ ed. Salvador de Bahía, Brasil. 143 p. 